Aus der staatl. Frauenklinik in Dresden

(Direktor: Ober-Med.-Rat Prof. Dr. E. Kehrer).

\title{
Die Grenzen der konservativen Geburtsleitung beim engen Becken.
}

Von

\section{Dr. Kurt Tittel,}

frūherem Assistenzarzt der Klinik, jetzigem Oberarzt der staatlichen Frauenklinik chemnitz.

Das Bestreben der modernen klinischen Geburtshilfe, bei einer möglichst geringen Gefährdung der Mutter auch das Leben des Kindes zu retten, musste noch vor wenigen Jahren zu einer Steigerung der Zahl grösserer geburtshilflicher Eingriffe führen, besonders in den Fällen, in denen infolge eines bestehenden Missverhältnisses zwischen Grösse des Kindes und Weite des mütterlichen Beckens auf einen glatten Geburtsverlauf nicht gerechnet werden konnte. Um diese Schwierigkeiten, die. dem Eintritt des kindlichen Kopfes in das Becken entgegenstanden, zu beseitigen oder zu vermindern und Mutter und Kind mit möglichster Sicherheit vor Schädigungen zu bewahren, kamen die Einleitung der künstlichen Frühgeburt, die prophylaktische Wendung, die hohe Zange, der Kaiserschnitt und die beckenerweiternden Methoden in ihren verschiedenen Modifikationen in Anwendung. Von den drei Faktoren, die wir als wesentlich für den Verlauf der Geburt anzusehen haben: Grösse des Kindes, Weite des Beckens, Stärke der Wehen, lässt sich der erste naturgemäss nur beeinflussen durch vorzeitige Unterbrechung der Schwangerschaft, der zweite nur durch Symphysiotomie oder Hebosteotomie, während alle drei Faktoren ausgeschaltet werden durch die Sectio eaesarea. Erst die Kenntnis der Einwirkung der Hypophysenpräparate auf die Uteruskontraktionen des Tieres (E. Kehrer 1908), die vermutlich darauf beruhende Einführung der Hypophysenpräparate in die praktische Geburtshilfe durch Foges und Hofstätter (1909) und die Feststellung der Indikationsgrenzen dieser neuen wertvollen Behandlungsweise (Hof bauer 1910) konnte in einer fast idealen Weise anch einen Einfluss auf die dritte Komponente, die Wehentätigkeit des gebärenden Uterus, gestatten, 
308 Tittel, Die Grenzen der konservativen Geburtsleitung beim engen Becken.

wodurch die Leitung der Geburt beim engen Becken sehr wesentlich erleichtert worden ist, ja, es wurde geradezu eine neue Aera herbeigeführt, die erst recht eigentlich der konservativen Geburtsleitung Berechtigung und Erfolg verlieh. Denn gerade die geringe Stärke oder die Seltenheit der Wehen waren es ja häufig gewesen, die auch schon bei mässigem Missverhältnis zwischen Kopf and Becken den Geburtshelfer zu einem aktiven Eingreifen veranlasst oder gezwungen hatten.

Heutzutage lässt sich die Behandlung der Geburt beim engen Becken, vorausgesetzt, dass das Missverhältnis desselben zum Kopf des Kindes nicht zu gross ist, in die Trias zusammenfassen: Pituitrin, Walcher'sche Hängelage, Hofmeier-Fritsch'sche Impression des Kopfes ins Becken, wobei nach den Anschauungen der Dresdener Klinik bei Erstgebärenden, zum mindesten bei enger Scheide, noch die Anwendung der Kolpeuryse etwa auf die Dauer von jeweils 6 Stunden in Anwendung zu kommen hat, um vorzeitigem Blasensprung vorzubeugen, die Weichteile zu erweitern und Wehen auch auf diesem reflektorischen Wege auszulösen. Bevor aber die Frau zur Geburt kommt, also in der letzten Zeit der Schwangerschaft, muss man sich bemühen, die Weite des Beckens durch instrumentelle innere Messung exakt festzustellen - denn die röntgenologische Pelvimetrie (E. Kehrer) ist nur in den ersten Monaten der Schwangerschaft möglich - und dabei gleichzeitig die Impression nach Peter Müller vorzunehmen, wozu freilich in der Regel Narkose erforderlich ist. Bei der Geburtsleitung aber ist zu berücksichtigen, dass Pituitrin nicht vorzeitig angewendet werden soll, da es im ersten Anfang der Eröffnungsperiode in der Regel weniger gut wirkt, wie am Ende derselben oder gar in der Austreibungszeit, und dass auch die Impression nach Hofmeier-Fritsch nur während der Wehe und nur dann ausgeführt werden darf, wenn die Fruchtblase gesprungen und der Muttermund zum mindesten kleinhandtellergross ist; denn sonst federt, wie Sellheim gezeigt hat, der Kopf nach jedem Impressionsversuch wieder zurück, während es andererseits in günstigen Fällen gelingt, den Kopf, zuweilen mit fühlbarem Ruck, in das Becken hineinzubringen.

Was die Indikationen und Kontraindikationen der abwartenden Geburtsbehandlung beim engen. Becken anbetrifft, so scheiden Eklampsie, schwere Nephritis und Árteriosklerose von vornherein aus, weil die Darreichung von Pituitrin wegen seiner blutdrucksteigernden Wirkung hier in der Regel nicht gestattet ist und es 
auch nicht erlaubt ist, eine Eklamptische den Umlagerungen auf dem Geburtsbett, welche die Walcher'sche Hängelage erfordert, oder dem energischen Druck der beiden Hände, der bei der Hofmeier-Fritsch'schen Impression angewendet werden muss, auszusetzen. Verboten ist auch weitere konservative Geburtsleitung bei Gefahr der Uterusruptur, bei Vorfall der Nabelschnur, von einzelnen Ausnahmen abgesehen, und im allgemeinen auch bei Verschlechterung der kindlichen Herztöne. Nicht in Frage kommen für die konservative Geburtsleitung auch Fälle mit stärkerer Beckenverengerung, falls man von vornherein annehmen muss, dass ein ausgetragenes Kind selbst bei kräftigster Wehentätigkeit und mit gut konfigurablem Schädel den Beckeneingang nicht passieren kann. Jeder Geburtshelfer aber wird Beweise dafür erbringen können, dass diese Abschätzung manchmal ausserordentlich schwer ist. Indiziert aber ist die konservative Geburtsleitung bei Schädellagen und Beckenverengerungen I., II. und III. Grades, falls also das mechanische Missverhältnis zwischen Becken und Kopf nicht so gross ist, daśs es nicht durch kräftige Wehentätigkeit bzw. Pituitrinverabreichung, Walcher'sche Hängelage und die erwähnte Impression überwunden werden kann. In diesen Fällen kann man durch richtige konservative Geburtsleitung häufig genug einen grösseren operativen Eingriff vermeiden. Vor allem wird sie auch da anzuwenden sein, wo die Kreissende eine grössere Operation ablehnt, oder wo sich der Geburtshelfer zu einer solchen nicht entschliessen kann. Ich denke hierbei an die Sectio caesarea classica bei nicht mehr einwandfrei aseptischem Genitale.

Die Erfolge der abwartenden Geburtsbehandlung beim engen Becken mögen an Hand der folgenden 33 Beobachtungen (vgl. Tabelle I), welche im wesentlichen aus den Jahren 1912 bis 1914 stammen und noch vor Ḱriegsbeginn abgeschlossen wurden, mitgeteilt werden.

Der Erfolg war insofern stets der erwünschte, als durch die oben erwähnte Trias, bei Erstgebärenden in der Regel auch unter gleichzeitiger Anwendung der Kólpeuryse, der Kopf des Kindes in das enge Becken eintrat und dann die unteren Beckenabschnitte leicht und schnell passierte. In den 33 Fällen machen davon nur 3 eine Ausnahme insofern, als hier wegen fötaler Indikation eine Beckenausgangszange notwendig geworden ist. In den 30 Fällen konnte durch die mit allen Mitteln geförderte konservative Geburtsleitung ein operativer Eingriff vermieden werden. Und die Becken- 


\begin{tabular}{|c|c|c|c|c|c|}
\hline Nr. & $\begin{array}{c}\text { Geb.- } \\
\text { Journ.- } \\
\text { Nr. }\end{array}$ & Name & Para & Becken & Frühere Geburten \\
\hline 1 & $\begin{array}{l}1512 \\
1912\end{array}$ & Ku., Antonie & II & Allgem. verengt, & 1. Geburt: Zange, Kind † p. p. \\
\hline 2 & $\begin{array}{l}1642 \\
1912\end{array}$ & P., Marie & II & $\begin{array}{l}\text { Pl.-rhachitisch, } \\
\text { C v. }=8,5 .\end{array}$ & 1. Geburt: Schwer, spontan. \\
\hline 3 & $\begin{array}{l}1649 \\
1912\end{array}$ & Fu., Martha & II & $\begin{array}{l}\text { Pl.-rhachitisch, } \\
\text { allg, verengt. }\end{array}$ & 1. Geburt: Spontan, $+\mathrm{n} .4$ Woch. \\
\hline 4 & $\begin{array}{l}1678 \\
1912\end{array}$ & Bl., Frieda & II & do. & Perforation in Klinik. \\
\hline 5 & $\begin{array}{l}1891 \\
1912\end{array}$ & Rü., Elsa . & II & Pl.-rhachitisch. & Spontan, $\dagger$ nach 6 Monaten. \\
\hline 6 & $\begin{array}{l}1982 \\
1912\end{array}$ & Kr., Agnes & $\mathbf{I}$ & $\begin{array}{l}\text { PI.-rhachitisch, } \\
\text { allg. verengt. }\end{array}$ & - \\
\hline 7 & $\begin{array}{l}2307 \\
1912\end{array}$ & Fi, Martha & IV & $\begin{array}{l}\text { Pl.-rhachitisch, } \\
\text { C. v. }=8,8 \text {. }\end{array}$ & $\begin{array}{l}\text { 1. lange Geb., spontan. 2. Per- } \\
\text { foration. 3. Perforation. }\end{array}$ \\
\hline 8 & $\begin{array}{l}2442 \\
1912\end{array}$ & D., Meta & VI & $\begin{array}{l}\text { Pl.-rhachitisch, } \\
\text { C. v. }=7,6 \text {. }\end{array}$ & $\begin{array}{l}\text { 1. Zange. 2. Wendung. 3. Wend. } \\
\text { Kind †p.p. 4. Wend. 5. Wend. } \\
\text { Perfor. d. nachfolg. Kopfes. }\end{array}$ \\
\hline 9 & $\begin{array}{l}2649 \\
1912\end{array}$ & T., Barbara & I & $\begin{array}{l}\text { Pl.-rhachitiseh, } \\
\text { allg. verengt. }\end{array}$ & - \\
\hline 10 & $\begin{array}{l}2869 \\
1912\end{array}$ & H., Agnete & $x$ & $\begin{array}{l}\text { Pl.-rhachitiseh, } \\
\text { C. } \nabla .=7,7\end{array}$ & $\begin{array}{l}\text { Kann keine genauen Angaben } \\
\text { machen. } 2 \text { Kinder leben. }\end{array}$ \\
\hline 11 & $\begin{array}{c}80 \\
1913\end{array}$ & Bo. & I & Pl.-rhachitisch. & - \\
\hline 12 & $\begin{array}{c}293 \\
1913\end{array}$ & He., Martha & VIII & do. & $\begin{array}{l}\text { 1. Zange. 2. Zwillinge. 3. Perfor. } \\
\text { 4. Spontane Frïhgeb. 5. bis } \\
\text { 7. Fehlgeburten. }\end{array}$ \\
\hline 13 & $\begin{array}{c}761 \\
1913\end{array}$ & N., Martha & VI & $\begin{array}{l}\text { Becken normal, } \\
\text { Missverhältnis. }\end{array}$ & 1.-4. Spontangeb. 5. Fehlgeb. \\
\hline 14 & $\begin{array}{l}1853 \\
1913\end{array}$ & Hel, Lisbeth & $\mathbf{I}$ & Pl.-rhachitisch. & 一 \\
\hline 15 & $\begin{array}{l}1892 \\
1913\end{array}$ & Fr., Gertrud & $\mathrm{I}$ & do. & - \\
\hline 16 & $\begin{array}{l}1930 \\
1913\end{array}$ & Bu., Wilhelm. & XII & do. & $10 \mathrm{mal}$ Wendung. 1 Fehlgeburt. \\
\hline 17 & $\begin{array}{l}1955 \\
1913\end{array}$ & Fi., Emma & $\mathbf{I}$ & do. $\%$ & 一 \\
\hline 18 & $\begin{array}{l}2053 \\
1913\end{array}$ & Dö., Ida & I & $\begin{array}{l}\text { Pl.-rhachitisch, } \\
\text { allg. verengt. }\end{array}$ & - \\
\hline 19 & $\begin{array}{l}2476 \\
1913\end{array}$ & Pa., Hilma & I & $\begin{array}{l}\text { Becken normal, } \\
\text { Missverhältnis. }\end{array}$ & - \\
\hline 20 & $\begin{array}{l}2553 \\
1913\end{array}$ & J., Martha & IV & Pl.-rhachitisch. & 3 Spontangeburten. \\
\hline 21 & $\begin{array}{l}2664 \\
1913\end{array}$ & Hä., Klara & VI & do. & 5 Spontangeburten. \\
\hline 22 & $\begin{array}{l}3146 \\
1913\end{array}$ & Ti., Martha & II & do. & I Spontangeburt. \\
\hline
\end{tabular}


be 11 e I.

\begin{tabular}{|c|c|c|c|c|}
\hline $\begin{array}{l}\text { Webonbeginn } \\
\text { ror der Walcher- } \\
\text { gehen Hänge- } \\
\text { lage + Impr. }\end{array}$ & $\begin{array}{c}\text { Eintritt bzw. } \\
\text { Geburt naeh } \\
\text { Walcher'scher } \\
\text { Hängelage } \\
\text { + Inpr. } \\
\end{array}$ & $\begin{array}{c}\text { Gewicht } \\
\text { u. Länge } \\
\text { des } \\
\text { Kindes }\end{array}$ & $\begin{array}{c}\text { Nabel- } \\
\text { schnur- } \\
\text { umschlin- } \\
\text { gung }\end{array}$ & $\begin{array}{l}\text { Bemerkungen } \\
\text { (Geburt und Verhalten, besonders } \\
\text { Gewicht, des Kindes) }\end{array}$ \\
\hline $16 \mathrm{~h} 15^{\prime}$ & $1 \mathrm{~h} \mathrm{45^{ \prime }}$ & $\begin{array}{c}4000 \\
54\end{array}$ & - & $\begin{array}{l}\text { Spontangeburt. Kind asphyktisch, lebt. } \\
15 . \mathrm{Tag}+30 \mathrm{~g} \text {. }\end{array}$ \\
\hline $1 \mathrm{Tag} 7 \mathrm{~h} 30^{\prime}$ & $50^{\prime}$ & $\begin{array}{c}3000 \\
50\end{array}$ & - & $\begin{array}{l}\text { Spontangeburt. Kind lebt. 9. Tag } \\
+120 \mathrm{~g} .\end{array}$ \\
\hline $14 \mathrm{~h}$ & $36^{\circ}$ & $\begin{array}{c}3050 \\
50\end{array}$ & - & $\begin{array}{l}\text { Geringe Hinterscheitelbeineinstellung. } \\
\text { Kräftige Wehen. Spontangeburt. Kind } \\
\text { lebt. 9. Tag - } 20 \mathrm{~g} \text {. }\end{array}$ \\
\hline $1 \operatorname{Tag} 3 \mathrm{~h} 36^{\circ}$ & $40^{\prime}$ & $\begin{array}{c}2750 \\
49\end{array}$ & - & $\begin{array}{l}\text { Kräftige Wehen. Spontangeburt. Kind } \\
\text { lebt. } 9 . \text { Tag }+160 \mathrm{~g} .\end{array}$ \\
\hline $15 \mathrm{~h} \mathrm{30}$ & $\begin{array}{l}10^{\prime} \\
\text { Geburt. }\end{array}$ & 3050 & $\begin{array}{l}1 \mathrm{mal} \text { um } \\
\text { den Hals }\end{array}$ & $\begin{array}{l}\text { Spontangeb., leicht asphyktisch, lebt. } \\
\text { 9. Tag - } 150 \mathrm{~g} .\end{array}$ \\
\hline $\begin{array}{c}2 \text { Tage } 20 \mathrm{~h} \\
16 \mathrm{~h} 45^{\prime}\end{array}$ & $\begin{array}{l}55^{\prime} \\
\text { Geburt. } \\
1 \mathrm{~h} \\
\text { Geburt. }\end{array}$ & $\begin{array}{c}3000 \\
49 \\
4000 \\
52\end{array}$ & - & $\begin{array}{l}\text { Hinterseheitelbeineinstellung. Spontan, } \\
\text { lebt. 9. Tag + } 100 \mathrm{~g} . \\
\text { Spontangeburt. Kind lebt. 9. Tag } \\
-70 \mathrm{~g} .\end{array}$ \\
\hline $18 \mathrm{~h}$ & $\begin{array}{l}2 \text { h } 20^{\circ} \\
\text { Geburt. }\end{array}$ & $\begin{array}{c}3150 \\
51\end{array}$ & - & Spontangeburt. Kind lebt. \\
\hline 2 Tage & $2 \mathrm{~h} 20^{\circ}$ & 3200 & - & Hinterscheitelbeineinstellung. Spontan- \\
\hline $21^{-} \mathrm{h}^{3} 30^{\prime}$ & $\begin{array}{l}\text { Geburt. } \\
1 \mathrm{~h} \\
\text { Geburt. }\end{array}$ & $\begin{array}{c}52 \\
3500 \\
53\end{array}$ & - & $\begin{array}{l}\text { geburt. Kind lebt. 9. Tag }-80 \mathrm{~g} \text {. } \\
\text { Starke Hinterscheitelbeineinstellung. } \\
\text { Kräftige Wehen. Spontangeb. Kind } \\
\text { lebt. } 16 \text { Tao }+{ }^{300} \mathrm{~g}\end{array}$ \\
\hline $1 \mathrm{Tag} 1 \mathrm{~h}$ & $2 \mathrm{~h} 30^{\prime}$ & $\begin{array}{c}3100 \\
53\end{array}$ & $\begin{array}{l}1 \mathrm{mal} \mathrm{um} \\
\text { den Hals }\end{array}$ & Spontan, totgeboren. \\
\hline 2 Tage $14 \mathrm{~h}$ & $1 \mathrm{~h} 15^{\prime}$ & $\begin{array}{c}3550 \\
50\end{array}$ & - & Spontangeburt, lebt. 9. Tag $-150 \mathrm{~g}$. \\
\hline $1 \mathrm{Tag} 3 \mathrm{~b}$ & $\begin{array}{c}50^{\prime} \\
\text { Geburt. }\end{array}$ & $\begin{array}{c}3200 \\
52\end{array}$ & - & $\begin{array}{l}\text { Beokenausgangszange wegen schlechter } \\
\text { Herztöne u. Mekoniumabgang. Kind } \\
\text { lebt. 9. Tag - } 60 \text { o. }\end{array}$ \\
\hline 2 Tage $2 \mathrm{~h}$ & $30^{\prime}$ & $\begin{array}{c}3300 \\
50\end{array}$ & - & $\begin{array}{l}\text { Spontan, leicht asphykt., lebt. Druck- } \\
\text { marken. Schiefer squamopar. Durch- } \\
\text { messer }=\end{array}$ \\
\hline & $\begin{array}{l}1 \mathrm{~h} \\
\text { Geburt. }\end{array}$ & $\begin{array}{c}3200 \\
51\end{array}$ & $\begin{array}{l}3 \text { mal lose } \\
\text { a. d. Hals }\end{array}$ & $\begin{array}{l}\text { Spontan, asphytitisch I0, lebt. } 9 . \text { Tag } \\
-270 \mathrm{~g} .\end{array}$ \\
\hline 2 Tage $16 \mathrm{~h}$ & $\begin{array}{l}4 \mathrm{~h} 45^{\prime} \\
\text { Geburt. }\end{array}$ & $\begin{array}{c}3700 \\
52\end{array}$ & - & $\begin{array}{l}\text { Spontangeburt, lebt. Druckmarken. } \\
9 . \text { Tag }+50 \mathrm{~g} .\end{array}$ \\
\hline $7 \mathrm{~b} \mathrm{10^{ \circ }}$ & $30^{4}$ & $\begin{array}{l}3400 \\
50\end{array}$ & $\begin{array}{l}1 \text { mal um } \\
\text { den Hals }\end{array}$ & $\begin{array}{l}\text { Spontangeburt. Kind lebt. 9. Tag } \\
-250 \mathrm{~g} .\end{array}$ \\
\hline 2 Tage $4 \mathrm{~h} 30^{\prime}$ & $30^{\circ}$ & $\begin{array}{c}2800 \\
49 \\
3200 \\
51\end{array}$ & - & $\begin{array}{l}\text { Hinterscheitelbeineinstellung. Spontan, } \\
\text { lebt. 9. Tag - } 350 \mathrm{~g} . \\
\text { Spontangeburt. Kind lebt. 9. Tag } \\
-110 \mathrm{~g} .\end{array}$ \\
\hline $\begin{array}{l}2 \text { Tage } \\
10 \mathrm{~h} 30^{\prime}\end{array}$ & $\begin{array}{l}3 \mathrm{~h} \cdot 30^{\prime} \\
\text { Geburt. }\end{array}$ & $\begin{array}{l}3000 \\
52\end{array}$ & - & $\begin{array}{l}\text { Hinterscheitelbeineinstellung. Ausser- } \\
\text { halb } 2 \text { mal Versuch d. hohen Zange. } \\
\text { Spontangeb., lebt. 9. Tag - } 130 \mathrm{~g} \text {. }\end{array}$ \\
\hline 2 Tage $2 \mathrm{~h}$ & $\begin{array}{l}45^{\prime} \\
\text { Geburt. }\end{array}$ & $\begin{array}{l}3300 \\
52\end{array}$ & - & $\begin{array}{l}\text { Hinterscheitelbeineinstellung. Spontan, } \\
\text { asphyktisch III } 0 \text {. } † \text { am 3. Tag. }\end{array}$ \\
\hline Tage & , & $\begin{array}{l}3350 \\
51\end{array}$ & 一. & $\begin{array}{l}\text { Hinterscheitelbeineinst. Ausserhalb } \\
\text { Zangenversuch. Spontangeburt, leicht } \\
\text { asphykt., lebt. 9. Tag }-400 \mathrm{~g} .\end{array}$ \\
\hline
\end{tabular}




\begin{tabular}{|c|c|c|c|c|c|}
\hline Nr. & $\begin{array}{c}\text { Geb.- } \\
\text { Journ.- } \\
\text { Nr. }\end{array}$ & Name & Para & Becken & Frühere Geburten \\
\hline 23 & $\begin{array}{c}171 \\
1914\end{array}$ & U., Anna & I & P1.-rhachitisch. & - \\
\hline 24 & $\begin{array}{l}203 \\
1914\end{array}$ & Gr., Eugenie & I & $\begin{array}{l}\text { Pl.-rhachitisch, } \\
\text { C. v. }=7,8 .\end{array}$ & - \\
\hline 25 & $\begin{array}{c}235 \\
1914\end{array}$ & Je., Alma & I & $\begin{array}{l}\text { Pl.-rhachitisch. } \\
\text { C. v. }=7,8 \text {. }\end{array}$ & - \\
\hline 26 & $\begin{array}{c}302 \\
1914\end{array}$ & Gö., Elsa & $I$ & Pl.-rhachit. II ${ }^{\circ}$ & - \\
\hline 27 & $\begin{array}{l}324 \\
1914\end{array}$ & Si., Agnes & I & $\begin{array}{l}\text { Pl.-rbachitisch, } \\
\text { C. v. }=8,0 \text {. }\end{array}$ & - \\
\hline 28 . & $\begin{array}{c}430 \\
1914\end{array}$ & Bi., Eilsa & I & $\begin{array}{l}\text { Pl. rhachitiseh, } \\
\text { C. } v .=9,1 \text {. }\end{array}$ & 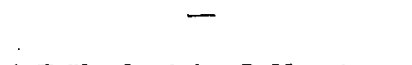 \\
\hline 29 & $\begin{array}{c}444 \\
1914\end{array}$ & Kra., Hermine & II & $\begin{array}{l}\text { Pl.-rhacbitisch, } \\
\text { C. } .=8,8 .\end{array}$ & 1 Frübgeburt im 7 . Monat. \\
\hline 30 & $\begin{array}{l}460 \\
1914\end{array}$ & Du. & I & $\begin{array}{l}\text { Pl.-rbachitisch, } \\
\text { allg. verengt II }\end{array}$ & - \\
\hline 31 & $\begin{array}{c}534 \\
1914\end{array}$ & Sp., Anna & II & Pl.-rhachit, II 0 . & 1. Zange, Kind + nach 3. Tagen. \\
\hline 32 & $\begin{array}{c}545 \\
1914\end{array}$ & La., Elsạ & I & Pl.-rhachitiseh. & - \\
\hline 33 & $\begin{array}{c}702 \\
1914\end{array}$ & Til., Emma & I & $\begin{array}{l}\text { Pl. rhachitisch, } \\
\text { C. v. }=9,3 \text {. }\end{array}$ & - \\
\hline
\end{tabular}

ausgangszange der letzten drei Geburtsbeobachtungen kann gegenüber der anderenfalls in Anwendung gekommenen hohen Zange nicht in die Wagschale fallen.

Interessant ist in den Fällen, in denen die Spontangeburt eines lebenden, ausgetragenen Kindes erfolgte, die Tatsache, dass häufig die früheren Geburten operativ beendet werden nmussten", und dass in einem Teil dieser Fälle, wie die Durchsicht der früheren Geburtsjournale ergeben hat, Gewicht und Länge bei der früheren Geburt nicht so gross waren als bei der jetzigen. So war in Nr. 16 der abwartenden Geburtsbehandlung nicht weniger wie $10 \mathrm{mal}$ eine prophylaktische Wendung vorausgegangen, während jetzt bei der 12. Geburt ein lebendes $3700 \mathrm{~g}$ schweres und $52 \mathrm{~cm}$ langes Kind, wenn auch mit Druckmarken am Schädel, geboren und lebend entlassen wurde. Bemerkenswert ist auch der Fall 7, in dem bei der ersten Geburt nach sehr langer Geburtsdauer ein vermutlich ziemlich kleines Kind tot spontan zur Welt kam und die zweite und dritte Entbindung durch Perforation des Kindes beendet werden 


\begin{tabular}{|c|c|c|c|c|}
\hline $\begin{array}{l}\text { Wehen beginn } \\
\text { vor der Waleher- } \\
\text { sehen Hänge- } \\
\text { lage + Impr. }\end{array}$ & $\begin{array}{l}\text { Eintritt bzw. } \\
\text { Geburt nach } \\
\text { Walcher'scher } \\
\text { Hängelage } \\
\text { + Impr. }\end{array}$ & $\left|\begin{array}{c}\text { Gewicht } \\
\text { u. Länge } \\
\text { des } \\
\text { Rindes }\end{array}\right|$ & $\begin{array}{c}\text { Nabel- } \\
\text { schnur- } \\
\text { umschlin- } \\
\text { gung }\end{array}$ & $\begin{array}{l}\text { Bemerkungen } \\
\text { (Geburt und Verhalten, besonders } \\
\text { Gewicht, des Kindes) }\end{array}$ \\
\hline 18 h $45^{\prime}$ & $45^{\circ}$ & $\begin{array}{c}3400 \\
50\end{array}$ & - & $\begin{array}{l}\text { Kräftige Wehen. Spontangeburt, lebt. } \\
9 . \text { Tag }-250 \mathrm{~g} \text {. Mutter im Wochen- } \\
\text { bett Zystitis. }\end{array}$ \\
\hline $16 \mathrm{~h} 30^{\prime}$ & $3 \mathrm{~h}$ & $\begin{array}{c}3300 \\
51\end{array}$ & - & $\begin{array}{l}\text { Zange aus B.A. weg. schlecht. Herztöne } \\
\text { u. Erschöpf. d. Frau. Kind asphykt., } \\
\text { wiederbel., † 3. Tg. Mutter i. Wochb. } \\
\text { fieberh. Zystitis, späterAdnexentzünd. }\end{array}$ \\
\hline $8 \mathrm{~h} 20^{\circ}$ & $1 \mathrm{~h} 40^{\prime}$ & $\begin{array}{c}3100 \\
50\end{array}$ & - & Spontan, asphykt., wiederbelebt. $\dagger$ am \\
\hline 2 Tage $2 \mathrm{~h}$ & $20^{\prime}$ & $\begin{array}{c}2700 \\
52\end{array}$ & - & $\begin{array}{l}\text { Zange aus B.A. wegen schlecht. Herz- } \\
\text { töne u. Mekoniumabgang. Kind lebt. }\end{array}$ \\
\hline $19 \mathrm{~h}$ & $25^{\prime}$ & $\begin{array}{c}2700 \\
45\end{array}$ & - & $\begin{array}{l}\text { Spontangeburt in tiefem Querstand. } \\
\text { Kind lebt. 9. Tag }-20 \mathrm{~g} \text {. }\end{array}$ \\
\hline $14 \mathrm{~h} \mathrm{30}$ & ? & $\begin{array}{c}3000 \\
50\end{array}$ & $\begin{array}{l}1 \text { mal um } \\
\text { den Hals }\end{array}$ & $\begin{array}{l}\text { Spontangeburt, leicht asphyktisch, lebt. } \\
\text { 9. Tag - 450 g. }\end{array}$ \\
\hline 1 Tag $8 \mathrm{~h}$ & $55^{\prime}$ & $\begin{array}{c}3100 \\
49\end{array}$ & do. & Spontangeburt, lebt. $9 . \mathrm{Tag}-100 \mathrm{~g}$. \\
\hline $1 \operatorname{Tag} 4 \mathrm{~h}$ & $2 \mathrm{~b} 30^{\circ}$ & $\begin{array}{c}2450 \\
49\end{array}$ & - & $\begin{array}{l}\text { Spontangeburt. Kind sehwer asphykt., } \\
\text { nieht wiederbelebt. }\end{array}$ \\
\hline $1 \mathrm{Tag} 12 \mathrm{~h}$ & 1 h $30^{\prime}$ & $\begin{array}{c}3200 \\
51\end{array}$ & - & Spontangeburt, lebt. 9. Tag $-160 \mathrm{~g}$. \\
\hline $1 \mathrm{Tag} 16 \mathrm{~h}$ & $36^{\prime}$ & $\begin{array}{c}2850 \\
40\end{array}$ & - & $\begin{array}{l}\text { Spontan, totgeboren. Zystitis im } \\
\text { Wochenbett. }\end{array}$ \\
\hline 2 Tage & ca. $24 \mathrm{~h}$ & $\begin{array}{c}3150 \\
52\end{array}$ & - & $\begin{array}{l}\text { Sectio verweigert, grosser hart. Schädel. } \\
\text { Spontan in tief. Querstand. †. Herz- } \\
\text { töne bis dicht vor der Geburt gut. } \\
\text { Geburtsdauer } 79 \text { Stunden. }\end{array}$ \\
\hline
\end{tabular}

musste, während jetzt bei abwartender Geburtsbehandlung die Spontangeburt eines nicht weniger als $4000 \mathrm{~g}$ schweren lebenden und lebend aus der Klinik entlassenen Kindes erfolgte; und der Fall 8 , in dem einer Zangenoperation und 4 maligen Wendungen mit fast immer toten Kindern die Spontangeburt eines $3150 \mathrm{~g}$ schweren Kindes folgte.

Aber diesem in bezug auf den Durchtritt des Kindes durch das Becken fast idealen Geburtsverlauf stehen doch gewisse Schädigungen von Mutter und Kind gegenüber.

Besteht schon durch eine lange Geburtsdauer, wie sie bei den Frauen mit dem in Sachsen so häufigen engen Becken in der Regel beobachtet wird, für die mütterlichen Weichteile die Gefahr der Quetschung und Nekrotisierung, welche zu Fieber in der Geburt oder im Wochenbett und möglicherweise zum Entstehen von Urinfisteln Veranlassung geben kann, so wächst diese Gefahr bei Anwendung einer etwas kraftrollen Methode, als welche die HofmeierFritsch'sche Impression im Verein mit der Walcher'schen Hänge- 
314 Tittel, Die Grenzen der konservativen Geburtsleitung beim engen Becken.

lage zu bezeichnen ist. Auch steht die Möglichkeit der Infektion zuweilen mit dem vorzeitigen Blasensprung in Zusammenhang, wodurch das Aufwandern von Keimen aus der Scheide in den Uterus möglich ist. Von unseren 33 Frauen haben trotz fast ausschliesslicher Rektaluntersuchung im Verlaufe der Geburt 8 bereits intra partum und $2 \mathrm{im}$ Wochenbett gefiebert. In einem Fall (24) schloss sich sogar eine langwierige fieberhafte Adnexentzündung an, die ätiologisch mit der langen Geburtsdauer vielleicht im Zusammenhang stehen könnte.

Ein zweiter Nachteil allzu konservativer Geburtsleitung liegt in dem langdauernden Druck des Kopfes auf die Weichteile. Wir haben unter den mitgeteilten Fällen, abgesehen von dem fast regelmässigen Oedem der Blasenschleimhaut, mehrfach Gelegenheit gehabt, zirkumskripte Nekrosen der hinteren Blasenwand cystoskopisch nachzuweisen. Vesiko-Vaginalfisteln oder Ureterfisteln haben wir glücklicherweise in diesen Fällen nicht beobachtet. Es ist bemerkenswert, dass die Blasennekrose gerade in den beiden Fällen 24 (Gross) und 25 (Jentsch) zustandekam, in denen die Impression des Kopfes lange Zeit vorgenommen werden musste. Aber diese Blasenveränderungen haben nur geringe Erscheinungen gemacht, und man kann in ihnen, solange keine Urinfisteln entstanden sind, eine Kontraindikation gegen die möglichst abwartende Geburtsleitung beim engen Becken wohl nicht erblicken.

Grösser als für die Mutter ist bei dieser Art der Geburtsleitung die Gefahr für das Leben des Kindes. Ein Blick auf unsere Zusammenstellung zeigt, dass unter den 33 Fällen 4 Kinder tot geboren wurden und 3 in den ersten Tagen nach der Geburt starben; das ist eine beträchtliche Mortalität von 21,2 pCt., und sie entspricht gewiss nicht ganz den Anforderungen der modernen Geburtshilfe. Bei den lebenden Kindern aber haben wir in der Regel eine verhältnismässig starke und andauernde Gewichtsabnahme festgestellt, so dass häufig noch am 9. Tag p. p., dem Zeitpunkt, zu dem die Mehrzahl der Kinder ihr Geburtsgewicht schon wieder erreicht haben, das Anfangsgewicht um 150, ja sogar bis $450 \mathrm{~g}$ unterschritten war. Hat die Schuld an diesen Gewichtsverlusten, an den häufigen Asphyxien gleich nach der Geburt und an der recht hohen Mortalität die konservative Geburtsleitung? Ist die Hofmeier-Fritsch-Impression zu gewalttätig ausgeführt worden oder sind die kindlichen Herztöne nicht genan genug bis zum erfolgten Kopfeintritt des Kindes kontrolliert worden? Diese letztere 
Tittel, Die Grenzen der konservativen Geburtsleitung beim engen Becken. 315

Frage glauben wir verneinen zu dürfen. Die Kontrolle der kindlichen Herztöne wurdè stets durch den Assistenzarzt und die Saalhebamme fast unausgesetzt vorgenommen, und auch bei den Impressionen waren wir bemüht, so vorsichtig wie es die Methode eben gestattet, vorzugehen.

Wie von vornherein erwartet wurde, gaben die Autopsiebefunde ${ }^{\mathrm{I}}$ ) eine klare Beantwortung der fraglichen Todesursachen (vgl. Tabelle II).

Auf Grund der klinischen Beobachtungen und Sektionsergebnisse sind in allen diesen 7 Fällen Blatungen in die Schädelhöhle bzw. Einrisse in Falx und Tentorium als Ursache des kindlichen Todes anzusehen. Die gleiche Schädigung wird aber auch in einigen anderen der 33 Fälle anzunehmen sein, wenn sie auch hier, ohne wesentliche Erscheinungen zu machen, zur Ausheilung kam. Dass diesen Gehirnblutungen eine weit grössere Bedeutung für die Mortalität der Neugeborenen zukommt als man früher glaubte, wissen wir jetzt durch zahlreiche Untersuchungen von Beneke, Ludwig Seitz, Esch u. A.

Die Blutungen, welche vorzugsweise in Einrissen in Falx und Tentorium begründet sind, entstehen beim Durchtritt des Kopfes besonders durch ein im Eingang enges Becken, zumal wenn derselbe schnell oder unter Zuhilfenahme einer stärkeren Kompression erfolgte. Eine solche aber ist bei der Hofmeier-Fritsch-Impression, bei welcher die vis a tergo, d. h. die Wehentätigkeit, durch eine von aussen wirkende Gewalt ersetzt wird, in der Regel nicht vermeidbar. Man kann dabei, falls man möglichst behutsam vorgeht und das Missverhältnis zwischen Kopf und Becken kein.allzugrosses ist, wohl den Kopf des Kindes ins Becken hineinbringen, ohne dass Gehirnblutungen eintreten, in der Regel aber ist eine Gewaltanwendung nötig, welche in der. Kompressibilität des kindlichen Schädels und der Architektur von Falx und Tentorium begründet ist. Diese Gefährdungsmöglichkeit des Kindes ist es nun, die in der letzten Zeit die Veranlassung gegeben hat, die Indikationsgrenzen der konservativen Geburtsleitung beim engen Becken etwas einzuschränken, die Hofmeier-Fritsch'sche Impression ... in

1) Die Sektionen wurden zum grösseren Teil im pathologisch-anatomischen Institut des Stadtkrankenhauses Johannstadt (Prosektor Prof. Geipel), zum kleineren Teil von dem wissenschaftlichen Assistenten der Frauenklinik Dr. Wil h. Lahm ausgeführt. Den Schädelsektionen wurde die Technik von Beneke zugrunde gelegt. 
316 Tittel, Die Grenzen der konservativen Geburtsleitung beim engen Becken.

Tabelle. II.

\begin{tabular}{|c|c|c|}
\hline $\begin{array}{c}\text { Name } \\
\text { und Fajl }\end{array}$ & Klinischer Befund und Diagnose & Sektion \\
\hline $\begin{array}{c}\text { Bo. } \\
\text { (Fall 11) }\end{array}$ & $\begin{array}{l}\text { Herztône bis kurz vor der Geburt gàt. } \\
\text { Kind mit schwachem Herzschlag ge- } \\
\text { boren. Nabelschnur } 1 \text { mal fest um den } \\
\text { Hals geschlungen, nicht wiederbelebt. } \\
\text { Scheitelbeine weit äbereinander ge- } \\
\text { schoben, starke Druckmarken v. Symphye } \\
\text { u. Promontorium. Offenbar Gehirnblutung. }\end{array}$ & Aus äusseren Gründen nicht gemacht. \\
\hline $\begin{array}{c}\mathrm{Hä.} \\
\text { (Fall 21) }\end{array}$ & $\begin{array}{l}\text { Kind bei Spontangeburt asphyktisch I0, } \\
\text { wiederbelebt. Druckmarke am rechten } \\
\text { Scheitelbein. Am 6. Tag Erscheinungen } \\
\text { von Hirndruck bzw. Gebirnblutung. Zu- } \\
\text { nebmende Zyanose und erschwerte At- } \\
\text { mung. Hemiparese d. recht.Körperbälfte. }\end{array}$ & $\begin{array}{l}\text { Ausgedehnte streifen- und flächenförmige } \\
\text { Blutungen im hinteren Teil der Falx } \\
\text { cerebri und dem Tentorium. Starke } \\
\text { Hyperämie des Sinus longitudinalis und } \\
\text { des transversus. Uebrige Sektion o. B. }\end{array}$ \\
\hline $\begin{array}{c}\text { Gr. } \\
\text { (Fall24) }\end{array}$ & $\begin{array}{l}\text { Zange am tiefstehenden Kopf. Kind } \\
\text { asphyktisch I0, wiederbelebt. Ersehei- } \\
\text { nungen von Gehirnbiutung. Am 3. Tag } \\
\text { zunehmende Zyanose, Atemstörungen } \\
\text { und Exitus. }\end{array}$ & $\begin{array}{l}\text { Impression d. linken Scheitelbeins. Durch- } \\
\text { blutung der Längsnaht. Einrisse in die } \\
\text { Falx und in die Schenkel des Tento- } \\
\text { riums. Bluterguss in die Seitenventrikel } \\
\text { und in den 3. Ventrikel. Subpleurale } \\
\text { und subperikardiale Blutungen. Häma- } \\
\text { tome auf den Herzklappen. }\end{array}$ \\
\hline $\begin{array}{c}\text { Je. } \\
\text { (Fall 25) }\end{array}$ & $\begin{array}{l}\text { Spontangeburt. Asphyxie Io. Nach ziem- } \\
\text { lich gutem Befinden am 2. Tag Krämpfe } \\
\text { und Tod an den Folgen einer Gehirn- } \\
\text { blatung. }\end{array}$ & $\begin{array}{l}\text { Kleine Blutungen in die weichen Hirn- } \\
\text { häute, besonders über dem Hinterhirn, } \\
\text { an den beiderseitigen Ansatzstellen des } \\
\text { Tentoriums, An diesern ist aud das } \\
\text { untere Blatt eingerissen. Gehirn blut- } \\
\text { reich. Subpleurale und subperikardiale } \\
\text { Blutungen; vereinzelte Blutungen in } \\
\text { den Lungen. }\end{array}$ \\
\hline $\begin{array}{c}\text { Du. } \\
\text { (Fall 30) }\end{array}$ & $\begin{array}{l}\text { Spontangeburt } 30 \text { Min. nach Eintritt des } \\
\text { Kopfes ins Becken. Asphyxie Il } 0 \text {, nicht } \\
\text { wiederbelebt. Starke Konfiguration des } \\
\text { Hinterbaupts, geringe des Vorderhaupts. }\end{array}$ & $\begin{array}{l}\text { Durchblutung der Koronarnaht. Kleiner } \\
\text { seitlicher Einriss im Tentorium. Ge- } \\
\text { hirnsubstanz stellenweise ausserordent- } \\
\text { lich blutreich, besonders im Corpus } \\
\text { striatum beiderseits. Stauungsorgane. }\end{array}$ \\
\hline $\begin{array}{c}\text { La. } \\
\text { (Fall 32) }\end{array}$ & $\begin{array}{l}\text { Spontangeburt, Asphyxie II }{ }^{0} \text {, Wiederbe- } \\
\text { lebungsversuche erfolglos. Stirnbein u. } \\
\text { Hinterhauptsbein unter die Parietalia } \\
\text { geschoben. }\end{array}$ & $\begin{array}{l}\text { Blutungen in die Koronarnaht und die } \\
\text { weichen Hirnhäute. Keine Einrisse in } \\
\text { Falx oder T'entórium. }\end{array}$ \\
\hline $\begin{array}{c}\text { Til. } \\
\text { (Fall 33) }\end{array}$ & $\begin{array}{l}\text { Lange Geburtsdauer (3 Tage } 7 \text { Stunden). } \\
\text { Wiederholte Kolpeuryse, Pituitrin, Wal- } \\
\text { cher, Hofmeier-Fritsch. Nach Eintritt } \\
\text { des Kopfes Herztöne bis kurz vor der } \\
\text { Geburt gut. Spontangeburt. Kind } \\
\text { asphyktisch, nieht wiederbelebt. }\end{array}$ & $\begin{array}{l}\text { Blutungen in die Umgebung der Koronar- } \\
\text { naht, in Falx und Tentorium. Aber } \\
\text { keine Tentoriumeinrisse. Zablreiche sub- } \\
\text { pleurale, subperikardiale und subendo- } \\
\text { kardialeBlutungen. An den Herzklappen } \\
\text { miliare Hämatome. }\end{array}$ \\
\hline
\end{tabular}

Walcher'scher Hängelage nur kurze Zeit und mit ganz besonderer Vorsicht zur Anwendung zu bringen und bei stärkerem Missverhältnis zwischen Kopf und Becken dieselbe abzulehnen. Bei Erstgebärenden lässt die Klinik jetzt die zervikale oder extraperitoneale Methode des Kaiserschnitts in Anwendung bringen, bei Mehrgebärenden aber die subkutane Symphysiotomie nach E. Kehrer, die im allgemeinen dié besten Resultate ergeben hat. 